\title{
Fad 24 Causes Hyperplasia in Adipose Tissue and Improves Glucose Metabolism
}

\author{
Yoshikazu Johmura, ${ }^{a}$ Kayoko Watanabe, ${ }^{a}$ Keishi Kishimoto, ${ }^{a}$ Takashi Ueda, ${ }^{b}$ Shoichi Shimada, ${ }^{b}$ \\ Shigehiro OsadA, ${ }^{a}$ Makoto NishizukA, ${ }^{a}$ and Masayoshi IMAgawA ${ }^{* a}$ \\ ${ }^{a}$ Department of Molecular Biology, Graduate School of Pharmaceutical Sciences, Nagoya City University; 3-1 Tanabe- \\ dori, Mizuho-ku, Nagoya, Aichi 467-8603, Japan: and ${ }^{b}$ Department of Neurobiology and Anatomy, Graduate School of \\ Medical Sciences, Nagoya City University; 1 Kawasumi, Mizuho-cho, Mizuho-ku, Nagoya, Aichi 467-8601, Japan. \\ Received May 17, 2009; accepted July 7, 2009; published online July 9, 2009
}

\begin{abstract}
We have previously reported that a novel gene, factor for adipocyte differentiation $(f a d) 24$, promotes adipogenesis in vitro. To examine the role of fad 24 in adipogenesis in vivo and the development of obesity, transgenic mice overexpressing $f a d 24$ were generated using mouse $f a d 24$ cDNA under the control of a chicken $\beta$-actin promoter and cytomegalovirus enhancer. The comparison of the ability of fibroblasts from $\mathrm{fad} 24$ transgenic embryos to differentiate into adipocytes with that of fibroblasts from wild-type embryos revealed that fad 24 overexpression promotes adipogenesis in embryonic fibroblasts. The weight and histology of white adipose tissues, and serum adipocytokine levels were compared between $f a d 24$ transgenic mice and wild-type mice, and we found that $f a d 24$ overexpression increased the number of smaller adipocytes, caused hyperplasia rather than hypertrophy in white adipose tissue and increased the serum adiponectin level in mice fed both normal chow and a high-fat diet. Glucose and insulin tolerance tests indicated that the activity for glucose metabolism is improved in fad 24 transgenic mice fed normal chow in comparison with that in wild-type mice. Our findings suggest that fad 24 is a positive regulator of adipogenesis in vivo. Moreover, the increase in the number of smaller adipocytes caused by the overexpression of $\mathrm{fad} 24$ appears to enhance glucose metabolic activity, perhaps by increasing the serum adiponectin level.
\end{abstract}

Key words obesity; adipogenesis; transgenic mouse; peroxisome proliferator-activated receptor $\gamma$; glucose metabolism; adiponectin

Obesity is a major risk factor for type 2 diabetes, hypertension, hyperlipidemia, and also arteriosclerosis. ${ }^{1)}$ The incidence of obesity has been increasing worldwide, and new approaches to treatment are desired. The increase in adipose tissue mass in obese individuals is thought to result from two distinct processes: the formation of new adipocytes from precursor cells (adipocyte differentiation) and an increase in adipocyte size due to fat storage (adipocyte hypertrophy). ${ }^{2,3)}$ Therefore, understanding the molecular mechanisms regulating these processes should provide valuable information in the fight to combat the worldwide epidemic of obesity.

Over the past two decades, the molecular regulation of adipocyte differentiation has been extensively characterized using the 3T3-L1 and 3T3-F442A preadipocyte cell lines. Notably, attention has centered on the roles of the nuclear receptor peroxisome proliferator-activated receptor $\gamma(\operatorname{PPAR} \gamma)$ and members of the CCAAT/enhancer-binding protein (C/EBP) family in the mid- and late stages of adipocyte differentiation. ${ }^{4)}$ These transcription factors are also known to influence adipocyte differentiation in vivo. Moreover, it is becoming clear that adipogenesis is regulated by an elaborate network of transcription factors, transcriptional cofactors, cell-cycle regulators, and signaling intermediates from numerous pathways. ${ }^{5,6}$ However, the earliest step in adipogenesis remains largely unknown, although others have recently reported regulators that are important during this period. ${ }^{7-10}$ )

Previously, we isolated 102 genes induced to express at the beginning of the differentiation of 3T3-L1 cells, using the polymerase chain reaction (PCR)-subtraction-cloning method. ${ }^{11,12)}$ One of them was a novel gene, factor for adipocyte differentiation (fad) 24. Gain-of-function and lossof-function experiments indicated that fad 24 promotes adipocyte differentiation in vitro. ${ }^{13)}$ More recently, we showed that FAD24 acts in concert with histone acetyltransferase binding to ORC1 (HBO1), a regulator of DNA replication, to promote adipogenesis by controlling a proliferating phase during adipocyte differentiation of 3T3-L1 cells, referred to as mitotic clonal expansion (MCE). ${ }^{14)}$

In this study, to determine the roles of $f a d 24$ in adipogenesis in vivo and the development of obesity, we created transgenic mice in which fad 24 is expressed under the control of a chicken $\beta$-actin promoter and cytomegalovirus enhancer. Our studies demonstrate that the ability of embryonic fibroblasts (EFs) from fad24 transgenic embryos to differentiate into adipocytes was superior to that of EFs from wild-type embryos. Fad 24 overexpression increased the number of smaller adipocytes in epididymal white adipose tissues (WAT) without a significant increase in adipose tissue mass. Consistent with the increase in the number of smaller adipocytes, the level of adiponectin, an adipocyte-derived hormone that can affect sensitivity to insulin, was elevated in fad 24 transgenic mice. Moreover, fad 24 transgenic mice had improved glucose metabolic activity. These results suggest that the overexpression of fad 24 promotes adipogenesis in vivo and subsequently improves insulin sensitivity by raising the level of adiponectin.

\section{MATERIALS AND METHODS}

Animal Studies Mice were housed in sterile cages in a barrier animal facility with a $12-\mathrm{h} / 12$-h light/dark cycle. They had access to food and water ad libitum, and were weighed every week. The animals were sacrificed by cervical dislocation, and blood was collected. Serum was stored at 
$-80^{\circ} \mathrm{C}$ after centrifugation. Subcutaneous and epididymal fat pads, liver, and kidney were dissected and weighed. Only males were used for experiments. All experiments using mice were carried out in accordance with guiding principles for the care and use of laboratory animals (Science and International Affairs Bureau of the Japanese Ministry of Education, Culture, Sports, Science and Technology) and also with the approval of the ethics committee of Nagoya City University.

Construction of a Plasmid and Generation of Transgenic Mice The mouse fad 24 open reading frame (ORF) was subcloned into the $E c o R I / M s c I$ site of a pCAGGS expression vector containing the chicken $\beta$-actin promoter and cytomegalovirus enhancer, $\beta$-actin intron and $\beta$-globin polyadenylation signal. ${ }^{15)}$ The fragment containing the promoter, ORF and poly-adenylation signal was excised with SalI and BamHI, gel-purified, and used as the transgene. Transgenic mouse lines were produced by injecting the purified transgene into BDF1 $\times$ BDF1 fertilized eggs (Japan SLC, Inc.). DNA-injected eggs were transplanted to ICR foster mothers, resulting in 68 newborns. We screened offspring for transgene transmission by Southern blot analyses of tail DNA using a cDNA probe to the $B g$ III/BglII site in fad 24 and PCR. The primers used for genotyping PCR were as follows: 5' primer, 5'-GGCTTCTGGCGTGTGACC-3', and 3' primer, 5'-TTCTCCCGAGCCTGAGGG-3'. The founder and trangenic descendants were bred onto a $\mathrm{C} 57 \mathrm{BL} / 6 \mathrm{~J}$ background for three generations. The F3 transgenic mice and their littermates were used in experiments. Transgenic mice served as heterozygotes.

Northern Blot Analysis The method used for the Northern blot analysis was described previously. ${ }^{13)}$ Briefly, total RNA was extracted with TriPure (Invitrogen) according to the manufacturer's instructions. The total RNA $(18 \mu \mathrm{g})$ from each tissue of each mouse was separated by electrophoresis on a formaldehyde-agarose gel and transferred to a nylon membrane, which was hybridized with a $\left[\alpha-{ }^{32} \mathrm{P}\right]$ deoxycytidine 5 '-triphosphate (dCTP)-labeled probe. The membrane was exposed to X-ray film for autoradiography.

Cell Culture and Adipocyte Differentiation We set up intercrosses of F2 fad 24 transgenic mice and C57BL/6J mice to recover embryos at day E14.5. We dissected embryos from the uterus and extraembryonic membranes. After the dissection of head and visceral organs for genotyping, embryos were minced into pieces, and trypsinized for $15 \mathrm{~min}$ at $37^{\circ} \mathrm{C}$. We stopped the reaction by adding Dulbecco's modified Eagle's medium (DMEM) supplemented with 10\% fetal bovine serum (FBS). Cell aggregates were resuspended by pipetting, plated on 100-mm culture dishes, and maintained in DMEM with $10 \% \mathrm{FBS}$ at $37^{\circ} \mathrm{C}$ in an atmosphere of $5 \%$ $\mathrm{CO}_{2}$. After $2 \mathrm{~d}$, we trypsinized EFs, replaced them on 12-well plates, and cultured them in the same medium. For the differentiation experiment, the medium was replaced with DMEM containing $10 \% \mathrm{FBS}, 10 \mu \mathrm{g} / \mathrm{ml}$ insulin, $0.5 \mathrm{~mm} 3$-isobutyl-1methylxantine and $1 \mathrm{~mm}$ dexamethasone $2 \mathrm{~d}$ post-confluent. After another $2 \mathrm{~d}$, the medium was changed to DMEM containing $10 \% \mathrm{FBS}$ and $5 \mu \mathrm{g} / \mathrm{ml}$ insulin, and replaced every $2 \mathrm{~d}$. To visualize lipid accumulation, cells were washed with phosphate-buffered saline (PBS), fixed with 4\% paraformaldehyde solution for $1 \mathrm{~h}$ and stained with Oil red $\mathrm{O}$ for $1 \mathrm{~h}$ using a $60: 40(\mathrm{v} / \mathrm{v})$ dilution in water of a $0.5 \%$ stock solution (in isopropanol). Cells were then washed twice with PBS and twice with water.

Real-Time Quantitative RT-PCR (Q-PCR) Total RNA was extracted with TriPure (Invitrogen) according to the manufacturer's instructions. The reverse transcription and QPCR were performed as previously described. ${ }^{13)}$ The predesigned primers and probe sets for fad24, ppar $\gamma$, fatty acid transporter, member 1 (fatpl) and 18S rRNA were obtained from Applied Biosystems.

Western Blot Analyses and Antibodies EFs were harvested before induction, and whole cell lysates were prepared with radio immunoprecipitation assay (RIPA) buffer (25 mM Tris- $\mathrm{HCl}, \mathrm{pH} 7.5,1 \%$ Triton $\mathrm{X}-100,0.1 \%$ sodium dodecyl sulfate (SDS), $0.5 \%$ sodium deoxycholate, $5 \mathrm{~mm}$ ethylenediamine tetraacetic acid (EDTA), and $150 \mathrm{~mm} \mathrm{NaCl}$ ) containing a protease inhibitor mixture. These lysates were resolved using SDS/PAGE, transferred to a polyvinylidene difluoride membrane, and probed using primary antibodies and secondary antibodies conjugated with alkaline phosphatase. Specific proteins were detected with 5-bromo-4-chloro-3-indolylphosphate/nitro blue tetrazolium (BCIP/NBT) phosphatase substrate (KPL). The antibody against mouse FAD24 was used as reported. ${ }^{14)}$ The antibody against $\beta$-actin (AC-15) was obtained from SIGMA.

Histological Analysis of WAT and Skeletal Muscle An epididymal fat pad and femoral muscle were removed from each mouse, and fixed in buffered 10\% formaldehyde. Fixed specimens were dehydrated and embedded in paraffin. The fat pad was then cut into $5-\mu \mathrm{m}$ sections at $50 \mu \mathrm{m}$ intervals, mounted on slides, and stained with hematoxylin and eosin. We calculated the adipose cell area with Scion Image software by manually tracing at least 400 adipocytes in three sections for each mouse. The total adipocyte number in WAT was determined by dividing the total WAT weight (milligrams) by the estimated mean adipocyte weight (milligrams). ${ }^{16)}$ The latter parameter was calculated by multiplying adipocyte density $(0.948 \mathrm{mg} / \mathrm{ml})$ by mean adipocyte volume (milliliters), which was determined from the average value of adipocyte diameter. The femoral muscle was cut into $6-\mu \mathrm{m}-$ thick longitudinal and cross sections and stained with hematoxylin and eosin.

Serum Adiponectin and Leptin Measurements Serum adiponectin and leptin concentrations were measured by the enzyme-linked immunosorbent assay (ELISA) using a mouse adiponectin ELISA kit (Otsuka Pharmaceutical Co.) and a mouse leptin ELISA kit (R\&D Systems), respectively.

Insulin Tolerance Test (ITT) Insulin tolerance was tested in mice fasted for $3 \mathrm{~h}$. The mice were intraperitoneally injected with human insulin at $1 \mathrm{mU} / \mathrm{g}$ body weight (Novo Nordisk A/S). Blood samples were drawn from a tail vein at specific points in time, and glucose was measured with Glucose C2 (Wako).

Glucose Tolerance Test (GTT) Glucose tolerance was tested in mice fasted for $16 \mathrm{~h}$. The mice were intraperitoneally injected with glucose at $2 \mathrm{mg} / \mathrm{g}$ body weight. Blood samples were drawn from the tail vein at the indicated times, and glucose was measured with Glucose C2 (Wako).

High-Fat Diet To study the effect of a high-fat diet, mice were fed a diet containing $32 \%$ calories from fat (CLEA Japan Inc.) from weaning (3 weeks of age) to the indicated weeks of age. 
Statistical Analysis Results were expressed as the mean \pm standard error, except in Fig. 2. Differences between groups were examined for statistical significance using Student's $t$ test.

\section{RESULTS}

Generation of Transgenic Mice Overexpressing Fad24 We generated transgenic mice overexpressing fad 24 ( $f a d 24$ transgenic mice) using the mouse fad 24 cDNA under the control of a chicken $\beta$-actin promoter and cytomegalovirus enhancer (Fig. 1a), which has the ability to express the transgene in various tissues including adipose tissues. ${ }^{15,17)}$ Sixty eight founder mice were obtained and screened for transgene transmission by Southern blot analyses and PCR of DNA. We identified 10 founder mice with various copy numbers of the transgene, and crossed them with $\mathrm{C} 57 \mathrm{BL} / 6 \mathrm{~J}$ mice to obtain F1 mice. Southern blot analyses of DNA from the F1 mice revealed that F1 mice with each copy number of the transgene were generated from 4 founder mice (line 2-5, 2-6, 3-7, 4-2) (data not shown). We also obtained F1 mice with moderate (line 4-3M) or high (line 4-3H) copy numbers of the transgene from line 4-3, and F1 mice with low (line 7$4 \mathrm{~L}$ ) or high (line $7-4 \mathrm{H}$ ) copy numbers of the transgene from line 7-4 (data not shown). We confirmed the transmission of the same copy numbers of the transgene to each F2 and F3 mouse, and finally obtained 8 lines of fad 24 transgenic mice. Northern blot analyses of various tissues from these transgenic mice and the wild-type mice demonstrated that 6 lines of fad 24 transgenic mice abundantly expressed fad 24 in

a

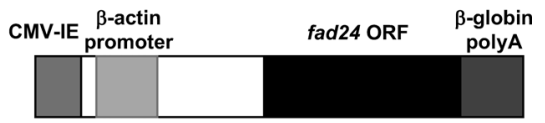

b

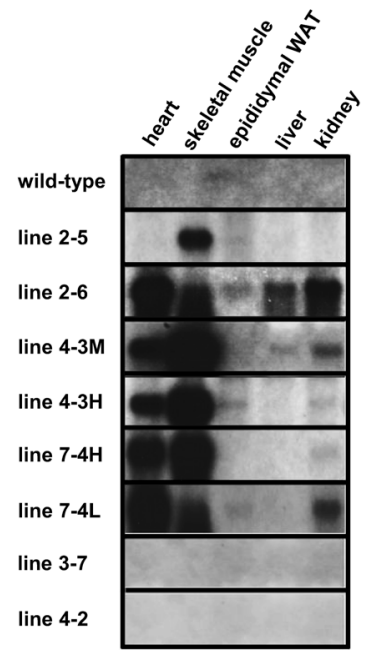

Fig. 1. Generation of Fad24 Transgenic Mice

(a) Schematic representation of the fad 24 transgene fragment used to generate fad 24 transgenic mice. The human cytomegalovirus immediate-early enhancer (CMV-IE) is followed by the chicken $\beta$-actin promoter with the first transcribed but untranslate exon, followed by the first chicken $\beta$-actin intron. The fad 24 cDNA is followed by the rabbit $\beta$-globin poly A donor. (b) Northern blot analysis of fad 24 mRNA in 8 lines of fad 24 transgenic mice and the wild-type mouse. Total RNA was prepared from heart, skeletal muscle, epididymal WAT, liver, and kidney from each F2 mouse. The total RNA $(18 \mu \mathrm{g})$ was loaded, and the filter was hybridized with the fad 24 probe. some tissues such as heart and skeletal muscle as compared to the wild-type mice (Fig. 1b). Especially in line 2-6 and line $4-3 \mathrm{H}$ of $f a d 24$ transgenic mice, $f a d 24$ was overexpressed in epididymal WAT relative to the wild-type animals and other lines of fad 24 transgenic mice. For subsequent analyses, we used line 2-6 and line 4-3H.

Effect of Fad24 Overexpression on Adipogenesis in EFs We first explored the roles of fad 24 in adipocyte differentiation per se by using EFs from line 2-6 of fad 24 transgenic embryos and the wild-type embryos. To examine the expression of $f a d 24$ in these EFs, we performed Q-PCR for fad 24 and found that fad 24 is overexpressed in EFs from fad 24 transgenic embryos relative to the wild-type embryos (Fig. 2a). Western blot analyses also showed the overexpression of FAD24 in EFs from fad24 transgenic embryos (Fig. 2b). Then, these cells were treated with adipogenic inducers. At $14 \mathrm{~d}$ post-induction, the cells were fixed and stained with Oil red O (Fig. 2c), and the amounts of triacylglycerol were determined (Fig. 2d). The number of Oil red O-stained cells and the accumulation of triacylglycerol were increased in EFs from fad 24 transgenic embryos relative to the wild-type embryos. Moreover, we determined the expression of ppar $\gamma$, a master regulator of adipogenesis, at $14 \mathrm{~d}$ post-induction by Q-PCR, and observed that the level of expression was increased in EFs from fad 24 transgenic embryos (Fig. 2e). Similar results were obtained using EFs from line 4-3H. a

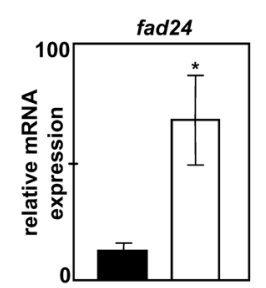

b

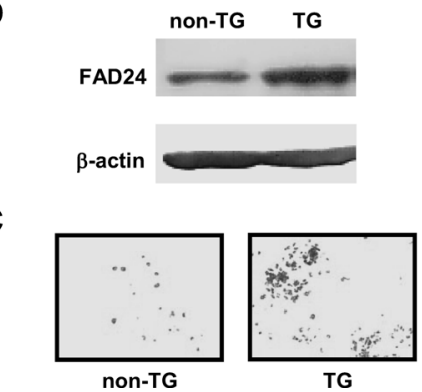

d

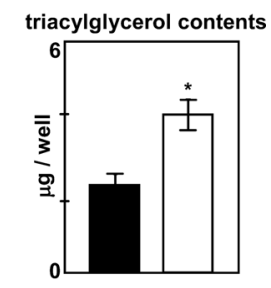

Fig. 2. Effect of Overexpression of Fad24 on Adipocyte Differentiation in EFs

(a) Q-PCR analyses of the expression of $f a d 24$. Total RNA was obtained from EFs from line 2-6 of fad 24 transgenic embryos (TG: white bar) and the wild-type embryos (non-TG: black bar) before induction. The expression level of fad 24 was determined by Q-PCR and normalized with $18 \mathrm{~S}$ rRNA expression. (b) Western blot analysis of the expression of FAD24. Whole cell lysates from the TG and non-TG EFs before induction were prepared, resolved by SDS/PAGE ( $8 \%$ gel), and detected by Western blotting with antibodies against FAD24 and $\beta$-actin. (c) Differentiation of EFs. The TG and non-TG EFs were treated with adipogenic inducers. At $14 \mathrm{~d}$ after induction, the cells were fixed and stained with Oil red O to detect oil droplets on 12-well plates. (d) Amounts of triacylglycerol were measured in the TG (white bar) and non-TG (black bar) EFs. (e) The expression of ppar $\gamma$, a master regulator of adipogenesis. Total RNA obtained from the TG (white bar) and non-TG (black bar) EFs at $14 \mathrm{~d}$ after induction was subjected to QPCR. The expression level was normalized with $18 \mathrm{~S}$ rRNA. Each value in a, d, and e represents the mean \pm standard deviation $(n=3)$. The asterisk and the double asterisks indicate significant differences when compared with the value for non-TG $(p<0.05$ and $p<0.005$, respectively). 
a

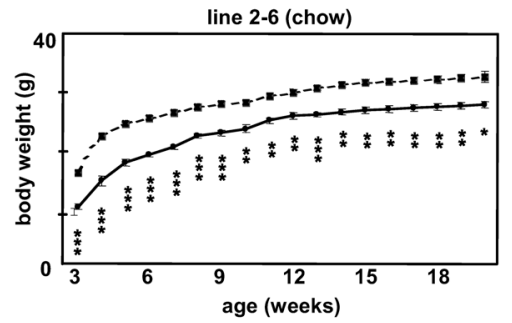

b

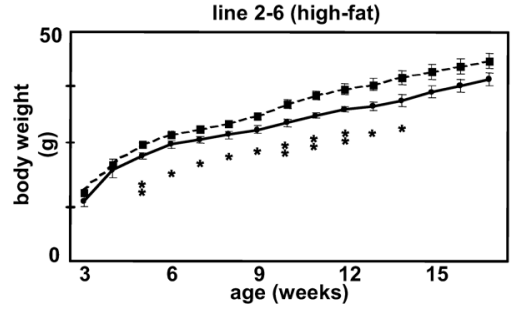

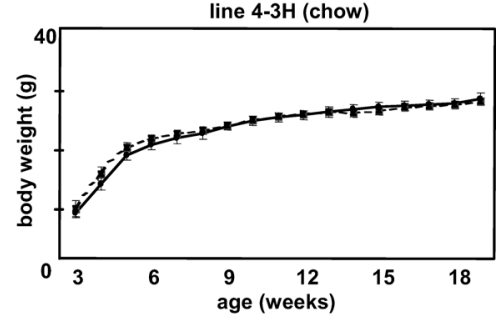

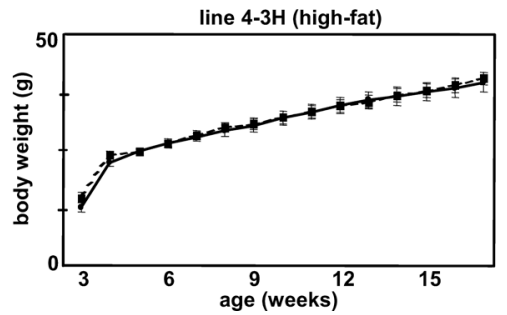

Fig. 3. Changes in Body Weights of Fad24 Transgenic Mice Fed Normal Chow or a High-Fat Diet

(a) Growth curves of mean body weight of line 2-6 of fad24 transgenic mice (left panel, solid line, $n=5$ ), the wild-type (left panel, dot line, $n=5$ ), line 4-3H of fad 24 transgenic mice (right panel, solid line, $n=7$ ) and the wild-type (right panel, dot line, $n=5$ ) under normal chow. (b) Growth curves of mean body weight of line 2 - 6 of fad 24 transgenic mice (left panel, solid line, $n=8$ ), the wild-type (left panel dot line, $n=10$ ), line $4-3 \mathrm{H}$ of $f a d 24$ transgenic mice (right panel, solid line, $n=4$ ) and the wild-type (right panel, dot line, $n=5)$ mice under a high-fat diet. The asterisk, the double asterisks, and the triple asterisks indicate significant differences when compared with the value for the wild-type mice $(p<0.05, p<0.01$, and $p<0.001$, respectively).

These results indicate that the overexpression of fad 24 promotes adipogenesis in EFs.

Effect of Fad24 Overexpression on Body Weight in Mice Fed Normal Chow To assess the roles of fad 24 in vivo, we fed normal chow to line 2-6 and line 4-3 of fad 24 transgenic mice and the wild-type mice. In line 2-6, we observed two groups of mice showing different phenotypes; At 3 weeks of age, one group had a decrease in body weight relative to the wild-type littermates as shown in Fig. 3a, and the other had no difference in body weight (data not shown). The former group was the majority. Moreover, subsequent analyses revealed the two groups to have similar phenotypes except for body weight. Therefore, we provide only the data obtained from the former group in this paper. As mentioned above, line 2-6 of fad 24 transgenic mice had a decrease in body weight at 3 weeks of age relative to the wild-type littermates, and maintained a decreased body weight until 20 weeks of age (Fig. 3a, left panel). On the other hand, line 4$3 \mathrm{H}$ of $\mathrm{fad} 24$ transgenic mice had no difference in body weight from 3 to 19 weeks of age relative to the wild-type littermates (Fig. 3a, right panel). Daily food and drink intakes did not differ between fad 24 transgenic mice and the wildtype mice in either line (data not shown).

Effect of Fad24 Overexpression on Adiposity and Serum Adipocytokine Levels in Mice Fed Normal Chow We measured the weights of epididymal and subcutaneous WAT, liver, and kidney from line 2-6 of fad 24 transgenic and wild-type mice. The absolute subcutaneous and epididymal WAT weights have no significant difference between wildtype mice and fad 24 transgenic mice. However, subcutaneous WAT weight per body weight was significantly heavier in fad24 transgenic mice than the wild-type littermates, but liver and kidney were not (Fig. 4a). Although statistically not significant, epididymal WAT weight per body weight was also greater in fad 24 transgenic mice. Next, we performed histological analyses of epididymal WAT to assess the size of adipocytes. Interestingly, microscopic observation revealed the number of adipocytes to be increased in the WAT from fad 24 transgenic mice relative to the wild-type littermates (Fig. 4b), although WAT weight was higher in fad24 transgenic mice. Measurements of size showed that the average size of adipocytes decreased and the population of smaller adipocytes increased in the WAT from fad 24 transgenic mice (Figs. 4c, d). Moreover, we found that the number of adipocytes was increased in fad 24 transgenic mice (Fig. 4e). These results suggest that the overexpression of fad 24 increased the number of smaller adipocytes, and caused hyperplasia in WAT.

Adipocytes secrete adipocytokines such as adiponectin and leptin which can affect sensitivity to insulin. ${ }^{18)}$ We measured adiponectin and leptin levels in serum, and found adiponectin, but not leptin, to be increased in fad 24 transgenic mice relative to the wild-type littermates (Figs. 4f, g). We also observed that the number of smaller adipocytes and adiponectin level in serum were increased in line $4-3 \mathrm{H}$ of fad 24 transgenic mice (data not shown). It is reported that promoting adipocyte differentiation and increasing the number of smaller adipocytes raises adiponectin levels. ${ }^{19)}$ Taken together, these results indicate that the increase in the number of smaller adipocytes caused by the overexpression of fad 24 elevated the adiponectin level in serum.

Effect of Fad24 Overexpression on Glucose Metabolism under Normal Chow Since fad 24 transgenic mice exhibited an increase in serum adiponectin levels as shown in Fig. 4, we carried out ITT and GTT to determine whether glucose metabolism was affected by the overexpression of fad24. ITT revealed that line 2-6 of fad 24 transgenic mice had a hypoglycemic response to insulin as compared to the wild-type littermates (Fig. 5a). GTT revealed that blood glucose levels at 30 and $60 \mathrm{~min}$ after the injection of glucose were lower in the transgenic mice (Fig. 5b). GTT and ITT showed that glucose metabolism was also improved in line $4-3 \mathrm{H}$ of $\mathrm{fad}_{24}$ 
a
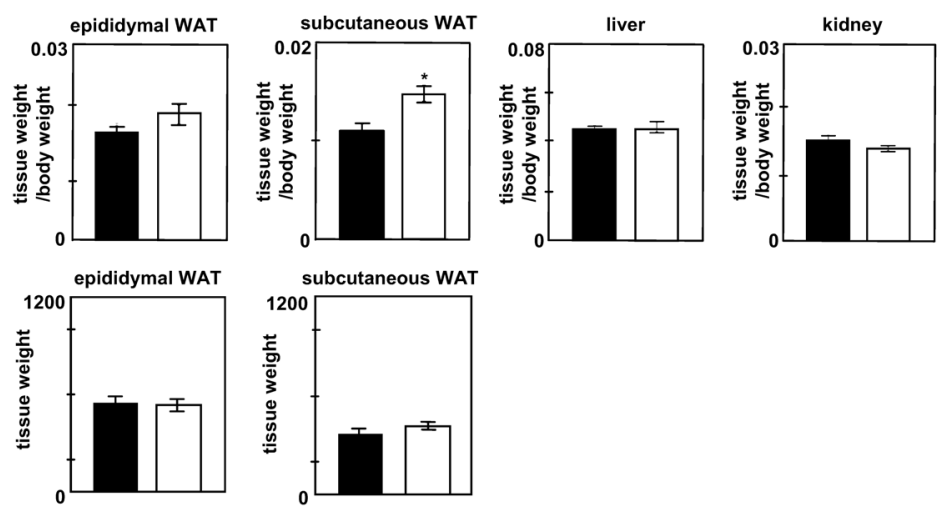

b
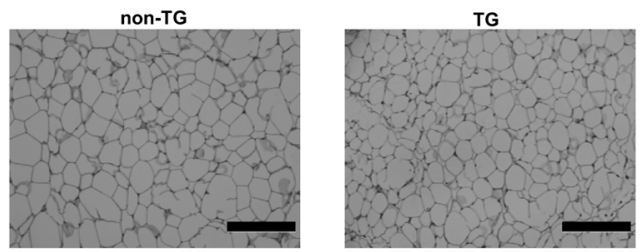

C

d
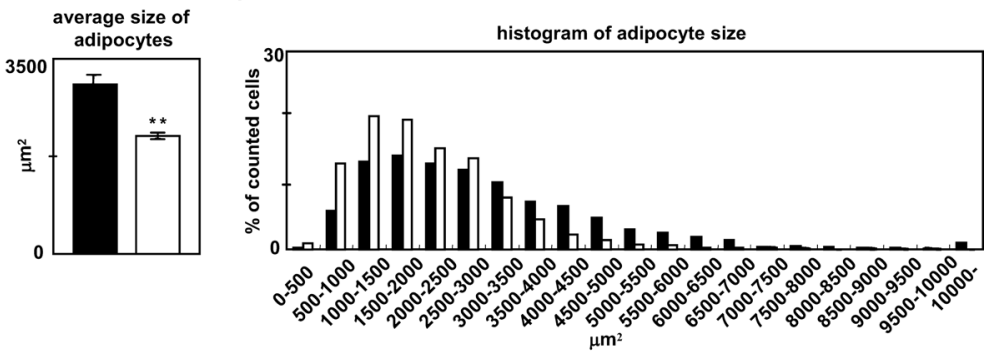

e

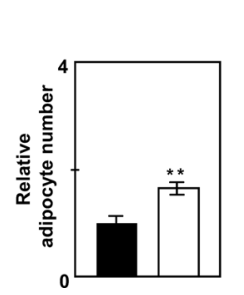

f
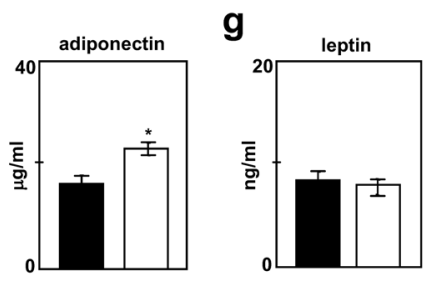

Fig. 4. Tissue Weights, Histological Analyses, and Serum Parameters of Fad24 Transgenic Mice Fed Normal Chow

(a) Tissue weights of line 2-6 of fad 24 transgenic mice and the wild-type mice. Fad 24 transgenic (TG: white bar, $n=4)$ and the wild-type (non-TG: black bar, $n=5$ ) mice were sacrificed and dissected at 23 weeks of age. Epididymal and subcutaneous WAT, liver, and kidney were weighed. The data were presented as the mean divided by body weight or absolute weights. (b) Histological appearance of epididymal WAT from line 2-6 of TG mice (right panel) and non-TG mice (left panel). Sections were stained with hematoxylin and eosin. Representative photomicrographs are presented. Scale bars indicate $200 \mu \mathrm{m}$. (c) Average size of adipocytes from epididymal WAT from line 2-6 of TG (white bar, $n=4$ ) and non-TG (black bar, $n=5$ ) mice. (d) Distribution of sizes of adipocytes. Sizes of individual adipocytes from line 2-6 of TG (white bar, $n=4$ ) and non-TG (black bar, $n=5$ ) mice were measured as described in Materials and Methods. The data represent the percentage of adipocytes of each size among the total cell number measured. (e) Relative number of adipocytes from line 2-6 of TG (white bar, $n=4$ ) and non-TG (black bar, $n=5$ ) mice. The data represent the mean normalized by the value for non-TG mice. (f) Serum adiponectin level. Serum adiponectin levels in line 2-6 of TG (white bar, $n=4$ ) and non-TG (black bar, $n=5$ ) mice were measured. (g) Serum leptin level. Serum leptin levels in line 2-6 of TG (white bar, $n=4$ ) and non-TG (black bar, $n=5$ ) mice were measured. The asterisk and the double asterisks indicate significant differences when compared with the value for nonTG mice ( $p<0.05$ and $p<0.005$, respectively).

transgenic mice (data not shown). These results suggest that the overexpression of fad 24 improved glucose metabolism.

Effect of Fad24 Overexpression on Body Weight in Mice on a High-Fat Diet To examine the effect of fad 24 overexpression on diet-induced obesity, we fed a high-fat diet to line 2-6 and line 4-3 of fad 24 transgenic mice and their wild-type littermates. As with the chow diet, line 2-6 showed a decrease in body weight relative to the wild-type mice (Fig. 3b, left panel), but line 4-3H did not (Fig. 3b, right panel).

Effect of Fad24 Overexpression on Adiposity and Serum Adipocytokine Levels in Mice Fed a High-Fat Diet Next, we measured the weights of epididymal and subcuta- neous WAT, liver, and kidney from line 2-6 of fad 24 transgenic mice and the wild-type mice fed a high-fat diet. Epididymal and subcutaneous WAT weights, but not liver and kidney weights, tended to increase in fad 24 transgenic mice relative to the wild-type littermates (Fig. 6a). Histological analyses of epididymal WAT revealed the population of smaller adipocytes to be increased in the fad 24 transgenic mice relative to the wild-type mice (Figs. $6 \mathrm{~b}-\mathrm{d}$ ). The number of adipocytes was also increased in fad 24 transgenic mice (Fig. 6e). The serum level of adiponectin, but not leptin, was also increased in fad 24 transgenic mice, although not significantly (Figs. 6f, g). Epididymal and subcutaneous 
a

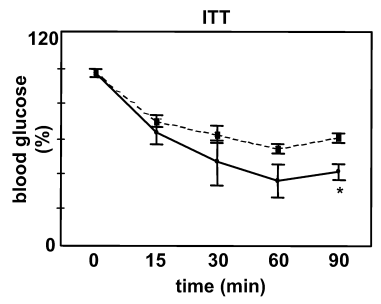

b

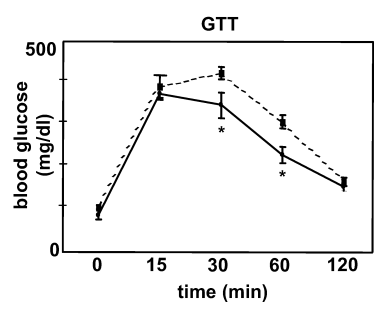

Fig. 5. ITT and GTT in Fad24 Transgenic Fed Normal Chow

(a) ITT. At 20 weeks of age, 3-h-fasted line 2-6 transgenic (solid line, $n=5$ ) and wild-type (dot line, $n=5$ ) mice were given an intraperitoneal injection of insulin ( $1 \mathrm{mU} / \mathrm{g}$ body weight). Blood samples were collected from the tail at the indicated time points and the concentration of glucose was analyzed. (b) GTT. At 22 weeks of age, 16 h-fasted line 2-6 transgenic (solid line, $n=5$ ) and wild-type (dot line, $n=5$ ) mice were given an intraperitoneal injection of glucose $(2 \mathrm{mg} / \mathrm{g}$ body weight). Blood samples were collected from the tail at the indicated time points and the concentration of glucose was analyzed. The asterisk indicates a significant difference when compared with the value for the wild-type mice $(p<0.05)$.

WAT weights and the serum level of adiponectin were also increased in line $4-3 \mathrm{H}$ of $\mathrm{fad} 24$ transgenic mice (data not shown). These results indicate that the overexpression of fad 24 increases the number of smaller adipocytes and subsequently elevates the serum adiponectin level under a high-fat diet.

Effect of Fad24 Overexpression on Glucose Metabolism in Mice on a High-Fat Diet We also performed ITT and GTT in line 2-6 of fad24 transgenic mice and the wild-type littermates fed a high-fat diet to examine the effect of diet-induced obesity on glucose metabolism. ITT revealed that line 2-6 had no significant hypoglycemic response to insulin as compared to the wild-type littermates (Fig. 7a). GTT showed that blood glucose levels after the injection of glucose did not differ between fad 24 transgenic mice and the wild-type littermates (Fig. 7b). Similar results were obtained using line 4$3 \mathrm{H}$ of fad 24 transgenic mice, namely GTT and ITT revealed that glucose metabolism was not improved in line $4-3 \mathrm{H}$ of fad 24 transgenic mice fed a high-fat diet (data not shown). These results suggest that the overexpression of fad $24 \mathrm{did}$ not improve glucose metabolism in diet-induced obesity.

Effect of Fad24 Overexpression on Skeletal Muscle Morphology and Expression of Fatty Acid Transporter As shown in Fig. 1b, fad 24 transgenic mice abundantly expressed fad 24 in skeletal muscle. To examine the effect of fad 24 overexpression on skeletal muscle morphology, histological analysis of skeletal muscle was performed. The femoral muscles of line 2-6 of fad 24 transgenic and wild type littermates were cut into longitudinal and cross sections and stained with hematoxylin and eosin. There were no striking morphologic changes including cell size and myofiber size in these skeletal muscles (Fig. 8). Similar results were obtained using line $4-3 \mathrm{H}$ of fad 24 transgenic mice (data not shown). Next, we examined the expression level of fatpl, a protein involved in flux of fatty acids into the cell. We did not observe significant differences in the expression level of fatpl in skeletal muscle between fad 24 transgenic and wild type littermates (data not shown).

\section{DISCUSSION}

In this study, we generated transgenic mice overexpressing fad 24 in various tissues to elucidate the roles of fad 24 in adi-

pogenesis in vivo and the development of obesity. The mouse adipocyte protein $2(\mathrm{aP} 2)$ promoter is often used to generate adipose tissue-specific transgenic mice, but is thought to express a transgene mainly in adipocytes into adipose tissues. ${ }^{20)}$ Our previous report revealed that the expression of fad 24 is transiently induced early in the adipocyte differentiation of 3T3-L1 cells, while these cells are fibroblastic. ${ }^{13)}$ We required the promoter to generate preadipocyte-specific transgenic mice. However, to our knowledge, such promoters have not been identified. To express fad 24 in preadipocytes, we used the pCAGGS expression vector containing a chicken $\beta$ actin promoter and cytomegalovirus enhancer, which has the ability to express the transgene in fibroblastic cells and various tissues. ${ }^{15,17)}$ Indeed, we were able to obtain transgenic mice which strongly express fad 24 in EFs and various tissues including WAT.

We examined the effect of fad 24 on adipocyte differentiation per se using EFs, and found that the overexpression promotes adipogenesis in EFs. At this time, how fad 24 promotes adipogenesis in EFs is not known. We previously revealed that fad24-overexpressing NIH-3T3 cells, non-differentiating cells, differentiated into adipocytes in the presence of a PPAR $\gamma$ agonist. ${ }^{13)}$ Since the level of ppar $\gamma$ expression was elevated in EFs from fad 24 transgenic embryos relative to the level in wild-type embryos, the function of fad 24 may be closely linked to the PPAR $\gamma$ pathway. Our recent report also indicated that fad 24 is deeply involved in MCE which appears to be necessary for optimal adipocyte differentiation of 3T3-L1 cells. ${ }^{14)}$ It is known that EFs as well as 3T3-L1 cells undergo MCE when treated with adipogenic inducers. ${ }^{21}$ Therefore, the overexpression of $f a d 24$ may promote MCE in EFs.

Interestingly, the population of smaller adipocytes was significantly increased in WAT from fad 24 transgenic mice relative to that from wild-type mice regardless of diet, although fad 24 transgenic mice showed a slight increase in adipose tissue mass. These results imply that the overexpression of fad24 caused hyperplasia rather than hypertrophy in WAT under both chow and high-fat diet conditions. Previously, it was reported that troglitazone, a PPAR $\gamma$ agonist, increased the number of smaller adipocytes without a change in adipose tissue mass. ${ }^{22,23)}$ The inhibition and depletion of Foxo1, a negative regulator of adipogenesis, are also known to increase the number of smaller adipocytes with a slight increase in adipose tissue mass. ${ }^{24,25)}$ The activation and overexpression of positive regulators of adipogenesis appear to increase the number of smaller adipocytes in adipose tissues but have little effect on adipose tissue mass. On the other hand, Shepherd et al. showed that the overexpression of glucose transporter 4 (GLUT4) selectively in adipose tissue results in increased fat mass by adipocyte hyperplasia. ${ }^{26)}$ However, it was also reported that adipose tissue-selective GLUT4 knockout mice have normal adipocyte size and number. ${ }^{27)}$ Further analyses will be required to reveal the detailed relationship between adipocyte hyperplasia and adipose mass.

In this study, the serum adiponectin level was elevated in fad 24 transgenic mice with the increase in the number of smaller adipocytes. Moreover, fad 24 overexpression improved glucose metabolism in mice fed normal chow. Since adiponectin is an insulin-sensitizing hormone, ${ }^{28,29)}$ the eleva- 
a
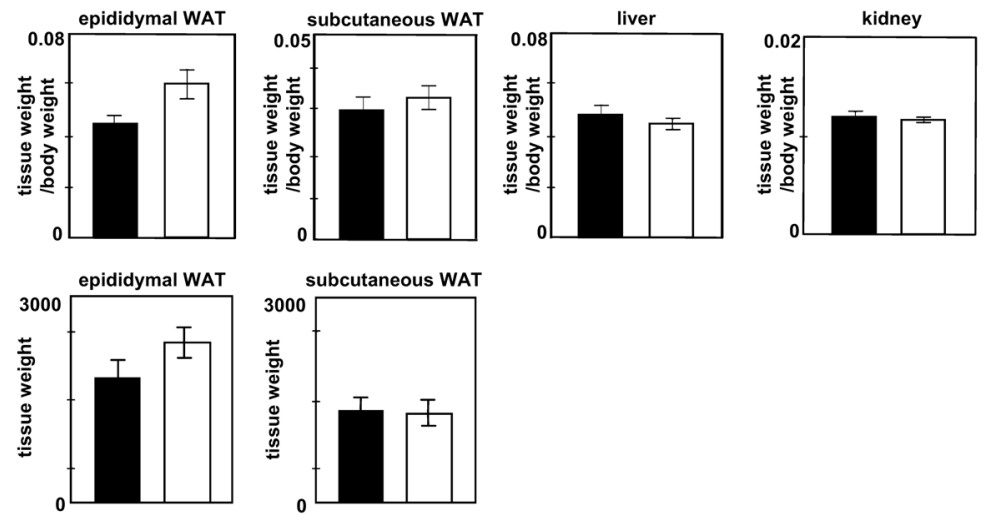

b
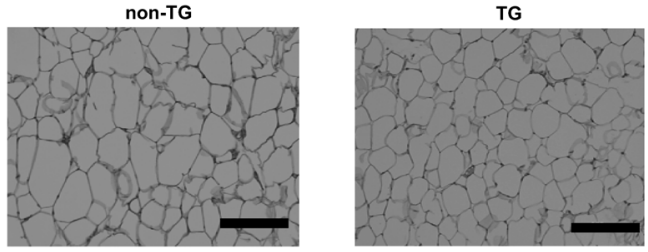

C

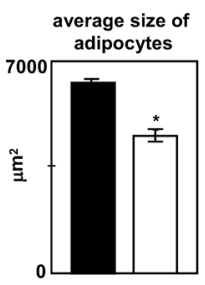

d

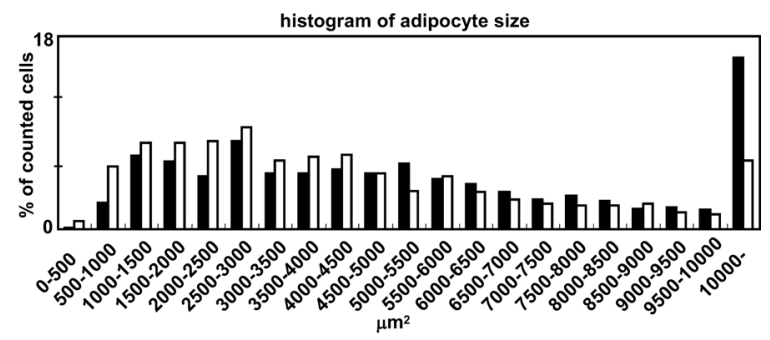

e

f

g
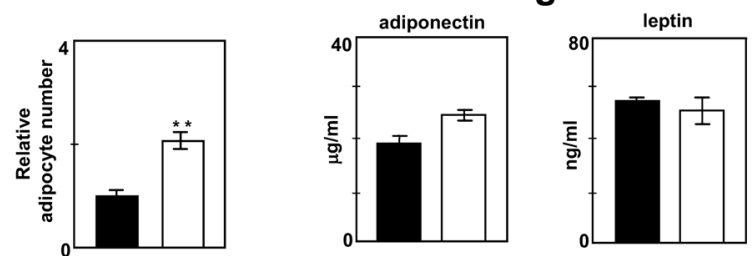

Fig. 6. Tissue Weights, Histological Analyses, and Serum Parameters of Fad24 Transgenic Mice Fed a High-Fat Diet

(a) Tissue weights of line 2-6 of $f a d 24$ transgenic mice and the wild-type mice. Fad24 transgenic (TG: white bar, $n=7$ ) and the wild-type (non-TG: black bar, $n=6$ ) mice were fed a high-fat diet from 3 weeks of age, sacrificed, and dissected at 15 weeks of age. Epididymal and subcutaneous WAT, liver, and kidney were weighed. The data were presented as the mean divided by body weight or absolute weights. (b) Histological appearance of epididymal WAT from line 2-6 of TG mice (right panel) and non-TG mice (left panel). Sections were stained with hematoxylin and eosin. Representative photomicrographs are presented. Scale bars indicate $200 \mu \mathrm{m}$. (c) Average size of adipocytes from epididymal WAT from line 2-6 of TG (white bar, $n=7$ ) and non-TG (black bar, $n=6$ ) mice. (d) Distribution of sizes of adipocytes. Sizes of individual adipocytes from line 2-6 of TG (white bar, $n=7$ ) and non-TG (black bar, $n=6$ ) mice were measured as described in Materials and Methods. The data represent the percentage of adipocytes of each size among the total cell number measured. (e) Relative number of adipocytes from line 2-6 of TG (white bar, $n=7$ ) and non-TG (black bar, $n=6$ ) mice. The data represent the mean normalized by the value for non-TG mice. (f) Serum adiponectin level. Serum adiponectin levels in line 2-6 of TG (white bar, $n=7$ ) and non-TG (black bar, $n=6$ ) mice were measured. (g) Serum leptin level. Serum leptin levels in line 2-6 of TG (white bar, $n=7$ ) and non-TG (black bar, $n=6$ ) mice were measured. The asterisk and the double asterisks indicate significant differences when compared with the value for the wild-type mice $(p<0.005$ and $p<0.0005$, respectively).

tion of the serum adiponectin level may improve glucose metabolism in fad 24 transgenic mice. Okuno et al. reported that troglitazone increases the number of smaller adipocytes and subsequently improves sensitivity to insulin by decreasing expression levels of tumor necrosis factor (TNF)- $\alpha$ and levels of plasma lipids. ${ }^{22}$ Further analyses including these factors will be required. Since the transgenic mice used in this study overexpress fad 24 in liver and skeletal muscle which are involved in glucose metabolism, it is also important to elucidate the roles of fad 24 in these tissues. On the other hand, under a high-fat diet, fad 24 transgenic mice exhibited an increase in serum adiponectin levels to the same extent as fad 24 transgenic mice fed normal chow, but showed no improvement in glucose metabolism. These results indicate that the elevation of the serum adiponectin level caused by the overexpression of fad 24 can not prevent the effect of dietinduced obesity on glucose metabolism.

As mentioned above, the overexpression of fad 24 in EF increased ppar $\gamma$ expression. It is known that ppar $\gamma$ regulates essential placental functions. ${ }^{30)}$ It is interesting to investigate whether fad 24 plays important roles in placental function. At least, we confirmed that fad 24 transgenic female mice have the capacity to fertilize. However, further analyses are required because it remains unknown whether fad 24 is overex- 
a

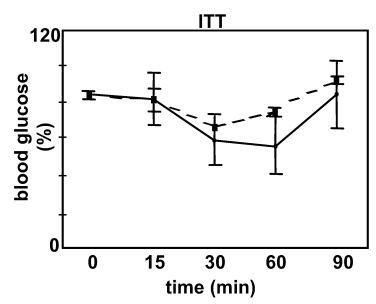

b

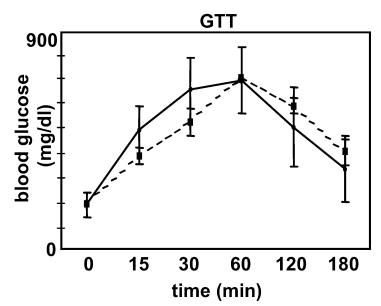

Fig. 7. ITT and GTT in Fad24 Transgenic Mice Fed a High-Fat Diet

(a) ITT. At 20 weeks of age, 3-h-fasted line 2-6 transgenic (solid line, $n=3$ ) and wild-type (dot line, $n=3$ ) mice fed a high-fat diet from 3 weeks of age were given an intraperitoneal injection of insulin $(1 \mathrm{mU} / \mathrm{g}$ body weight). Blood samples were collected from the tail at the indicated time points and the concentration of glucose was analyzed. (b) GTT. At 22 weeks of age, 16-h-fasted line 2-6 transgenic (solid line, $n=3$ ) and wild-type (dot line, $n=3$ ) mice fed a high-fat diet from 3 weeks of age were given an intraperitoneal injection of glucose $(2 \mathrm{mg} / \mathrm{g}$ body weight). Blood samples were collected from the tail at the indicated time points and the concentration of glucose was analyzed.
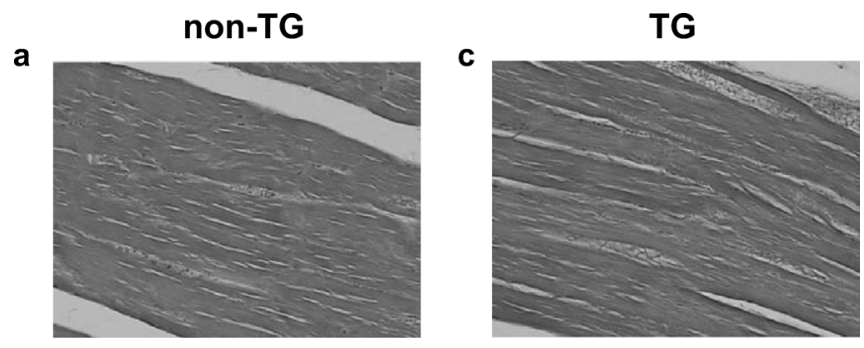

b
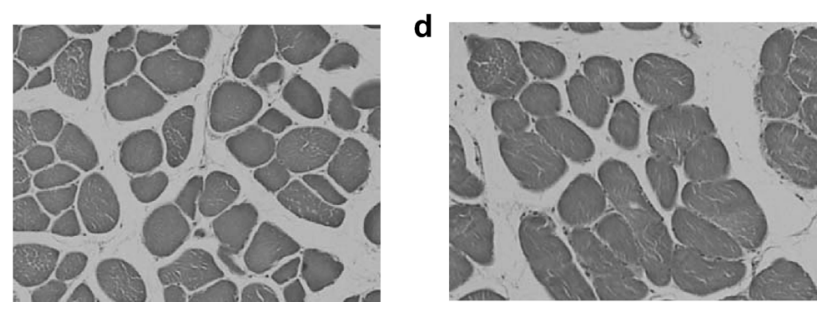

Fig. 8. Histological Analysis of Skeletal Muscle of Fad24 Transgenic Mice

The skeletal muscles of line 2-6 of TG mice (c, d) and non-TG mice (a, b) were removed from each mouse at 64 weeks of age. The skeletal muscles embedded in paraffin were cut into longitudinal (a, c) and cross (b, d) sections and stained with hematoxylin and eosin.

pressed in placenta in this mouse.

The two lines of fad 24 transgenic mice used in this study showed different phenotypes for body weight when fed both normal chow and a high-fat diet. At present, this cause remains unknown. We measured the weights of several tissues including liver, kidney, heart and skeletal muscle. However, these data show no significant difference between wild-type mice and fad 24 transgenic mice. Considering that the transgene were expressed in various tissues, $f a d 24$ overexpression may slightly decrease various tissue weights and subsequent decrease body weight. More analyses of all tissues in detail will be required. Since line 2-6 expresses fad 24 in many tissues relative to line $4-3 \mathrm{H}$, the overexpression of fad 24 may affect tissues other than adipose tissue only in line 2-6. In contrast, the two lines showed similar results in the number of smaller adipocytes and glucose metabolism. These characteristics do not appear to be affected by the phenotypes for body weight. Since we utilized global promoter, the expression levels of the transgene were considerably higher in tissues other than adipose tissue in the line 2-6, raising a possi-

bility of suspicion that significantly lean body weight might had an impact on adipocyte morphology, adiponectin levels and glucose metabolism. However, the line $4-3 \mathrm{H}$ of $\mathrm{fad} 24$ transgenic mice had no difference in body weight, and the number of smaller adipocytes and adiponectin levels was increased in these mice. Glucose metabolism was also improved in these mice, suggesting that significantly lean body weight had a minor effect on adipocyte morphology, adiponectin levels and glucose metabolism in fad 24 transgenic mice.

Both line 2-6 and line 4-3H of fad 24 transgenic mice abundantly expressed fad 24 in skeletal muscle. However, in this study, the histological analysis of the skeletal muscle from fad 24 transgenic mice showed no obvious differences compared with those of wild-type littermates. Although our previous reports indicated that human fad 24 was highly expressed in skeletal muscle, the function of fad 24 in skeletal muscle was not clear yet. ${ }^{13)}$ Considering fad24 is strongly overexpressed in skeletal muscle, further analyses including the measures of glucose and lipid metabolite levels in skeletal muscle are definitely needed.

In conclusion, the overexpression of $\mathrm{fad} 24$ promoted adipocyte differentiation in EFs and increased the number of smaller adipocytes and caused hyperplasia in white adipose tissue, suggesting that fad 24 is a positive regulator of adipogenesis in vivo. Subsequently, the overexpression appears to improve glucose metabolism with an elevated serum adiponectin level. Consistent with other reports, ${ }^{22-25}$ ) these results raise the possibility that the promotion of adipogenesis increases the number of smaller adipocytes without a significant increase in adipose tissue mass and leads to the alleviation of insulin resistance. Further characterization of fad 24 including analyses of $f a d 24$ knockout mice should help us to understand the roles of adipogenesis in obesity and metabolic diseases.

Acknowledgements We thank Dr. Jun-ichi Miyazaki (Osaka University) for generously providing the pCAGGS expression plasmid. We also thank Dr. Masaru Okabe (Osaka University) for discussing the generation of $\mathrm{fad}^{24}$ transgenic mice. This work was supported in part by Grants from the Ministry of Education, Culture, Sports, Science and Technology (MEXT) of Japan, and Japan Society for the Promotion of Science (JSPS).

\section{REFERENCES}

1) Kopelman P. G., Nature (London), 404, 635-643 (2000).

2) Hausman D. B., DiGirolamo M., Bartness T. J., Hausman G. J., Martin R. J., Obes. Rev., 2, 239-254 (2001).

3) Bjorntorp P., Horm. Metab. Res., 4, $77-83$ (1976).

4) Rosen E. D., Walkey C. J., Puigserver P., Spiegelman B. M., Genes Dev., 14, 1293-1307 (2000).

5) Farmer S. R., Cell Metab., 4, 263 -273 (2006).

6) Rosen E. D., MacDougald O. A., Nat. Rev. Mol. Cell Biol., 7, 885896 (2006).

7) Ross E. S., Hemati N., Longo K. A., Bennett C. N., Lucas P. C., Erickson R. L., MacDougald O. A., Science, 289, 950—953 (2000).

8) Tong Q., Dalgin G., Xu H., Ting C. N., Leiden J. M., Hotamisligil G. S., Science, 290, 134-138 (2000).

9) Oishi Y., Manabe I., Tobe K., Tsushima K., Shindo T., Fujiu K., Nishimura G., Maemura K., Yamauchi T., Kubota N., Suzuki R., Kitamura T., Akira S., Kadowaki T., Nagai R., Cell Metab., 1, 27-39 (2005). 
10) Chen Z., Torrens J. I., Anand A., Spiegelman B. M., Friedman J. M., Cell Metab., 1, 93-106 (2005).

11) Imagawa M., Tsuchiya T., Nishihara T., Biochem. Biophys. Res. Commun., 254, 299-305 (1999).

12) Nishizuka M., Tsuchiya T., Nishihara T., Imagawa M., Biochem. J., 361, 629-633 (2002).

13) Tominaga K., Johmura Y., Nishizuka M., Imagawa M., J. Cell Sci., 117, 6217-6226 (2004).

14) Johmura Y., Osada S., Nishizuka M., Imagawa M., J. Biol. Chem., 283, $2265-2274$ (2008).

15) Niwa H., Yamamura K., Miyazaki J., Gene, 108, 193-200 (1991).

16) Sakai T., Sakaue H., Nakamura T., Okada M., Matsuki Y., Watanabe E., Hiramatsu R., Nakayama K., Nakayama K. I., Kasuga M., J. Biol. Chem., 282, 2038-2046 (2007).

17) Okabe M., Ikawa M., Kominami K., Nakanishi T., Nishimune Y., FEBS Lett., 407, 313-319 (1997).

18) Rosen E. D., Spiegelman B. M., Nature (London), 444, 847-853 (2006).

19) Yamauchi T., Kamon J., Waki H., Murakami K., Motojima K., Komeda K., Ide T., Kubota N., Terauchi Y., Tobe K., Miki H., Tsuchida A., Akanuma Y., Nagai R., Kimura S., Kadowaki T., J. Biol. Chem., 276, 41245-41254 (2001).

20) Kamei N., Tobe K., Suzuki R., Ohsugi M., Watanabe T., Kubota N., Ohtsuka-Kowatari N., Kumagai K., Sakamoto K., Kobayashi M., Yamauchi T., Ueki K., Oishi Y., Nishimura S., Manabe I., Hashimoto H., Ohnishi Y., Ogata H., Tokuyama K., Tsunoda M., Ide T., Murakami K., Nagai R., Kadowaki T., J. Biol. Chem., 281, 26602-26614 (2006).

21) Tang Q. Q., Otto T. C., Lane M. D., Proc. Natl. Acad. Sci. U.S.A., 100,
$850-855$ (2003).

22) Okuno A., Tamemoto H., Tobe K., Ueki K., Mori Y., Iwamoto K., Umesono K., Akanuma Y., Fujiwara T., Horikoshi H., Yazaki Y., Kadowaki T., J. Clin. Invest., 101, 1354-1361 (1998).

23) Kubota N., Terauchi Y., Miki H., Tamemoto H., Yamauchi T., Komeda K., Satoh S., Nakano R., Ishii C., Sugiyama T., Eto K., Tsubamoto Y., Okuno A., Murakami K., Sekihara H., Hasegawa G., Naito M., Toyoshima Y., Tanaka S., Shiota K., Kitamura T., Fujita T., Ezaki O., Aizawa S., Kadowaki T., Mol. Cell, 4, 597-609 (1999).

24) Nakae J., Kitamura T., Kitamura Y., Biggs W. H., Arden K. C., Accili D., Dev. Cell, 4, 119-129 (2003).

25) Nakae J., Cao Y., Oki M., Orba Y., Sawa H., Kiyonari H., Iskandar K., Suga K., Lombes M., Hayashi Y., Diabetes, 57, 563-576 (2008).

26) Shepherd P. R., Gnudi L., Tozzo E., Billis J. L., Kahn B. B., J. Biol. Chem., 268, 22243-22246 (1993).

27) Abel E. D., Peroni O., Kim J. K., Kim Y. B., Boss O., Hadro E., Minnemann T., Shulman G. I., Kahn B. B., Nature (London), 409, 729733 (2001).

28) Yamauchi T., Kamon J., Waki H., Terauchi Y., Kubota N., Hara K., Mori Y., Ide T., Murakami K., Tsuboyama-Kasaoka N., Ezaki O., Akanuma Y., Gavrilova O., Vinson C., Reitman M. L., Kagechika H., Shudo K., Yoda M., Nakano Y., Tobe K., Nagai R., Kimura S., Tomita M., Froguel P., Kadowaki T., Nat. Med., 7, 941—946 (2001).

29) Berg A. H., Combs T. P., Du X., Brownlee M., Scherer P. E., Nat. Med., 7, 947-953 (2001).

30) Barak Y., Nelson M. C., Ong E. S., Jones Y. Z., Ruiz-Lozano P., Chien K. R., Koder A., Evans R. M., Mol. Cell, 4, 585-595 (1999). 